\title{
Co-delivery of miR-29b and germacrone based on cyclic RGD-modified nanoparticles for liver fibrosis therapy
}

\author{
De $\mathrm{Ji}^{1^{* *}}$ (1), Qiaohan Wang ${ }^{1 \dagger}$, Qi Zhao ${ }^{2,3}$, Huangjin Tong ${ }^{4}$, Mengting Yu' ${ }^{1}$, Meng Wang ${ }^{1}$, Tulin $\mathrm{Lu}^{1 *}$ \\ and Chengxi Jiang $2,3^{*}$
}

\begin{abstract}
Hepatic stellate cells (HSCs) were activated and secreted excessive amounts of extracellular matrix (ECM) proteins during pathogenetic progress of liver fibrosis. Germacrone (GMO) and miR-29b can play an important role in inhibiting growth of HSCs and production of type I collagen. GMO and miR-29b were co-encapsulated into nanoparticles (NPs) based on poly(ethylene glycol)-block-poly(lactide-co-glycolide) (PEG-PLGA). Then, NPs were modified with cyclic RGD peptides (cRGDfK). CRGDfK is an effective ligand to bind integrin $a_{v} \beta_{3}$ and increase the targeting ability for fibrotic liver. GMO- and miR-29b-loaded NPs exhibited great cytotoxicity to activated HSCs and significantly inhibited production of type I collagen. Liver fibrosis model of mice was induced by administration of carbon tetrachloride. Great targeting ability was achieved in liver fibrotic mice treated with CRGD-modified NPs. Significant ant-fibrotic effects have been presented based on hematoxylin and eosin (H\&E), Masson and Sirius Red staining results of liver tissues collected from mice treated with drug-loaded NPs. All these results indicate GMO- and miR-29b-loaded cRGD-modified NPs have the potential for clinical use to treat liver fibrosis.
\end{abstract}

Keywords: Hepatic stellate cells, Germacrone, miR-29b, cRGDfK, Liver fibrosis therapy

\section{Introduction}

Liver fibrosis has been serious health problem with high worldwide dominance and poor prognosis [1-4]. Liver fibrosis is a responsive process of virtually all forms of chronic liver injury, including hepatitis infection, excess alcohol consumption, and metabolic disorders [5-7]. With the deepening of liver fibrosis, it may result in cirrhosis, organ failure, and even hepatocellular carcinoma [8]. In this process, hepatic stellate cells (HSCs) were activated by factors released from damaged hepatocytes

\footnotetext{
*Correspondence: jidenucm@163.com; It1209@163.com; jiangchengxi@126. com

†De Ji, Qiaohan Wang contributed equally to this work

${ }^{1}$ School of Pharmacy, Nanjing University of Chinese Medicine, Nanjing 210023, China

2 Molecular Pharmacology Research Center, School of Pharmaceutical Science, Wenzhou Medical University, Wenzhou 325035, China

Full list of author information is available at the end of the article
}

and secreted excessive extracellular matrix (ECM). ECM contains different components, including collagen, proteoglycans, fibronectin, and hyaluronic acid, dominated by collagen I $[9,10]$. Thus, activated HSCs become important targets of anti-fibrotic therapy and the therapeutic effect can be reflected by the level of collagen I. After decades of efforts for basic and clinical trials, several antifibrotic agents are currently in clinical trials [11]. However, no drugs have yet emerged as effective anti-fibrotic agents, because medicinal candidates cannot specifically target HSCs and are frequently toxic to parenchymal cells [12]. Thus, there is still an urgent need to find new drugs and develop effective anti-fibrotic therapies [4].

Germacrone (GMO) is one of the main bioactive components in the traditional Chinese medicine Rhizoma curcuma [13]. Fufang Biejia Ruangan Pill (FFBJ) has been approved by the China State Food and Drug Administration to use as the first anti-fibrosis drug [14]. 
Noteworthily, GMO is an important component and has been demonstrated inducing G2/M cell cycle arrest and apoptosis through inhibition of JAK2/STAT3 signal pathway against human hepatoma cell lines [13]. However, the anti-fibrosis effect of GMO has not been reported yet. In this study, GMO was selected as the potential drug and encapsulated into NPs to evaluate its anti-fibrosis effect.

MicroRNAs (miRNAs) are receiving increasing attention for the management of different diseases. miRNAs are endogenous small noncoding RNAs and act as the regulator for RNA expression [11]. miRNAs exhibit great safety and potential for disease treatment due to their endogenous nature and biological functions. Besides, miRNAs have already been exploited for the treatment of liver fibrosis [15-17]. miRNAs play a crucial role in the initiation and progression of liver fibrosis. As reported, miR-29b family is significantly down-regulated during the activation of HSCs and progression of liver fibrosis [18]. miR-29b targets several pro-fibrotic genes like collagen type I \& IV, c-MYC, platelets derived growth factor beta (PDGF- $\beta$ ), and PI3K/AKT through their $3^{\prime}$-UTR regions [19]. Notably, miR-29b down-regulates phosphorylation of protein kinase $B(A K B)$ which is involved in growth and proliferation, adhesion, migration, and collagen production by HSCs. Transforming growth factor beta 1 (TGF- $\beta 1$ ) up-regulated in liver fibrosis and known to down-regulate miR-29b through SMAD3 pathway. Therefore, liver fibrosis can possibly be attenuated by restoring intracellular levels of miR-29b to inhibit fibrogenic signaling at multiple levels and expression of ECM proteins.

In recent years, drug delivery systems have attracted increasing attention because of their unique advantages, like tissue or cell targeting [20-22], combined therapy $[23,24]$ and imaging [25-28]. Drug delivery system strategies have showed great potential for cancer treatment by improving traditional chemotherapy $[29,30]$ and incorporating novel therapy technology [31-34]. Although some drug delivery systems have been developed for the treatment of liver fibrosis (Table 1) [10, 12, 35-41], most of the work involved complex material synthesis or nanocarrier construction, which makes them difficult for massive generation and hence reduces their potential for clinical application. Besides, few new therapeutic agents were studied as the potential options of liver fibrosis therapy. Therefore, it is really still worth exploring new anti-fibrotic agents and paying more attention for clinical application. In this study, GMO was studied as a potential anti-fibrotic agent for the first time and co-loaded with miR-29b into NPs based on commercial block polymers poly(ethylene glycol)-block-poly(lactide-co-glycolide) (PEG-PLGA) to establish a drug delivery system for liver fibrosis therapy. To enhance the targeting ability, cRGDfK was connected with the carboxyl groups of NPs by amido bonds. Drug-loaded NPs were characterized by particle size, Zeta potential, drug loading, miRNA complexation as well as stability and transfection efficiency, and evaluated for treating liver fibrosis. Our results suggest a new approach for co-delivery of miRNAs and small hydrophobic molecules by biodegradable polymeric NPs.

\section{Materials and methods \\ Materials}

$\mathrm{PLGA}_{10 \mathrm{k}}-\mathrm{PEG}_{5 \mathrm{k}}-\mathrm{COOH}$ was purchased from Huateng Pharma. Germacrone (GMO) was brought from I\&K Scientific (China). miR-29b was supplied by Guangzhou RiboBio Co.,LTD. (China, 98\%). Indocyanine green (ICG) was purchased from Tianjing HEOWNS Biochemical Technology Co., Ltd. (China, 98\%). cRGDfK was brought from Hangzhou Peptide Biochem Co.,Ltd. (China, 97\%). Poly(vinyl alcohol) (PVA, MW: 30000) was brought from Aladdin Biochemical Technology Co., Ltd. (China). N-Hydroxysuccinimide (NHS, China, 99\%) and 1-(3-dimethylaminopropyl)-3-ethylcarbodiimide hydrochloride (EDC; China, 99\%) were obtained from Sigma-Aldrich. 3-(4, 5-Dimethylthiazol-2-yl)-2,

Table 1 Recent development of drug delivery systems for liver fibrosis therapy

\begin{tabular}{llll}
\hline Nanocarriers & Targeted molecules & Drugs & Reference \\
\hline liposomes & Vitamin A & Imatinib & {$[10](2017)$} \\
PLGA-PSPE-PEG NPS & Vitamin A & Silibinin \& siCol1a1 & {$[12](2018)$} \\
CaP@BSA NPs & $/$ & TSG-6 & {$[35](2020)$} \\
HA nanomicelles & Hyaluronic acid & Silibinin & {$[36](2019)$} \\
PEG-PLGA NPs & Vitamin A & Nilotinib & {$[37](2019)$} \\
CS nanomicelles & Chondroitin sulfate & Doxorubicin \& retinoic acid & {$[38](2019)$} \\
Lipid nanoparticles & Vitamin A & siCol1a1 \& siTIMP-1 & {$[39](2020)$} \\
Liposomes & CRGDyK & Vismodegib & {$[40](2019)$} \\
Polymeric micelles & $/$ & Polydatin & {$[41](2020)$} \\
\hline
\end{tabular}


5-diphenyltetrazolium bromide (MTT, China, 99\%) and dihydrochloride (DAPI, China, 99\%) was purchased from Sigma-Aldrich (Shanghai) Trading Co., Ltd. BCA protein assay kit was brought from Beyotime Biotechnology Co., Ltd. Carbon tetrachloride $\left(\mathrm{CCl}_{4}\right.$, China, AR) was purchased from Energy Chemical. Organic solvents including chloroform and dimethyl sulfoxide (DMSO) were brought from Greagent (China, AR).

\section{Preparation and modification of NPs}

The GMO- and miR-29b-loaded PEG-PLGA-COOH NPs (G/R-NPs) were prepared using the oil-in-water $(\mathrm{O} / \mathrm{W})$ emulsion method [42]. Briefly, PLGA-PEG$\mathrm{COOH}(12.5 \mathrm{mg})$ was dissolved in $0.25 \mathrm{~mL}$ chloroform and mixed with $2.3 \mathrm{mg}$ GMO in $15 \mu \mathrm{L}$ chloroform under ultrasound. Then $15 \mu \mathrm{L}$ miR-29b was added dropwise into the organic phase and mixed by sonication at $0{ }^{\circ} \mathrm{C}$ for $2 \mathrm{~min}$. The solution was further dropwise added into $1.5 \mathrm{~mL}$ of $1 \%(\mathrm{wt} / \mathrm{v})$ PVA and mixed by sonication for another $2 \mathrm{~min}$. At last, $0.3 \%$ PVA $(25 \mathrm{~mL})$ was added and the mixture was stirred at room temperature overnight to evaporate the organic solvent. The obtained emulsion was washed by ultrafiltration for three times (MW $100 \mathrm{kD}$ ) at $4{ }^{\circ} \mathrm{C}$ and $1.25 \mathrm{~mL}$ PBS (pH 7.4) was added to absorb the NPs.

To modify cRGD onto the NPs, EDC $(0.25 \mathrm{mg})$ and NHS $(1.25 \mathrm{mg}$ ) were mixed with NPs suspension and stirred for $30 \mathrm{~min}$. cRGD $(1.25 \mathrm{mg})$ was added and the reaction were stirred at $4{ }^{\circ} \mathrm{C}$ overnight. The cRGD-modified G/R-NPs (G/R-RGD-NPs) were purified by ultrafiltration (MWCO 5000) for three times at $10000 \mathrm{rpm}$ to remove EDC, NHS and unconjugated cRGD. Single drug loaded NPs were prepared and modified by the same method, abbreviated as G-RGD-NPs and R-RGD-NPs, and used in the following experiments.

To visually demonstrate the enhanced cellular uptake and targeting ability of cRGD-modified NPs, ICG-loaded NPs based on PEG-PLGA-COOH were prepared and modified with cRGD by the same procedure, abbreviated as ICG-NPs and ICG-RGD-NPs, respectively.

The colloidal stability of cRGD-modified PEG-PLGA NPs was evaluated by DLS in different conditions: water, saline and culture medium containing 10\% fetal bovine serum (FBS). Briefly, NPs were incubated in different conditions and shaken slightly at $37^{\circ} \mathrm{C}$ for a week. The particle size of NPs was measured by DLS every day. The experiment was performed for three times.

\section{Characterization of NPs}

The size distribution and Zeta potential of blank NPs, G/R-NPs and G/R-RGD-NPs were measured by dynamic light scattering (DLS). The morphology of NPs was observed by transmission electron Microscopy (TEM,
H7100). The TEM sample was prepared by the typical procedure as reported [43]. In brief, $10 \mu \mathrm{L}$ NPs suspension was dropped onto carbon-coated copper grids. After stained for $5 \mathrm{~min}$, negative staining with $2 \%$ phosphotungstic acid was used to enhance the contrast. After drying for $1 \mathrm{~h}$ at room temperature, NPs images were observed under TEM.

\section{Determination of drug loading content (DLC) and efficiency (DLE)}

DLC of GMO in G/R-RGD-NPs was determined by high performance liquid chromatography (HPLC, Shimadzu, Japan). In brief, G/R-RGD-NPs were dissolved in methanol. Sonication was performed to destroy NPs. After filtration with $0.22 \mu \mathrm{m}$ filter, the filtrate was analyzed by HPLC at excitation wavelength $210 \mathrm{~nm}$. Mobile phase comprised of acetonitrile/phosphoric acid solution $(50 / 50, \mathrm{v} / \mathrm{v}, \mathrm{pH} 3.24)$ with the flow rate of $1.0 \mathrm{~mL} / \mathrm{min}$.

The DLC of miR-29b was measured spectrophotometrically. Briefly, after miR-29b-loaded NPs were prepared, the suspension was centrifuged and the liquid supernatant was collected to measure the amount of free miR$29 \mathrm{~b}$. The amount of encapsulated miR-29b is equal to the total amount of input minus the amount of free miR-29b. DLC and DLE were calculated as follows:

$$
\begin{aligned}
& D L C \%=\frac{\text { Amount of drugs entrapped in NPs }}{\text { Initial amount of drug added }} \times 100 \% \\
& D L E \%=\frac{\text { Amount of drugs entrapped in NPs }}{\text { Total amount of NPs }} \times 100 \%
\end{aligned}
$$

\section{Drug release}

The release profiles of G/R-RGD-NPs were investigated in PBS at $37{ }^{\circ} \mathrm{C}$. Briefly, G/R-RGD-NPs $(1 \mathrm{mg} / \mathrm{mL})$ were added into a dialysis tube (MW 3500) and immersed in $10 \mathrm{~mL}$ mixture of PBS with $1 \%$ SDS (pH 7.4): methanol $(60 / 40, v / v)$ in a brown bottle [44]. The bottle was then placed at $37^{\circ} \mathrm{C}$ with mild shaking. At the predetermined time points during incubation, $1 \mathrm{~mL}$ release medium was taken out to measure drug content and the same volume of fresh release medium was added. The release medium was lyophilized and re-dissolved in methanol and filtrated through $0.22 \mu \mathrm{m}$ filter before measurement.

\section{Cell culture}

HSCs cells were cultured in DMEM supplemented with $10 \%$ fetal bovine serum (FBS), 100 units $/ \mathrm{mL}$ penicillin and $100 \mu \mathrm{g} / \mathrm{mL}$ streptomycin. LO2 cells were maintained in RPMI-1640 media containing 10\% FBS, 100 units $/ \mathrm{mL}$ penicillin and $100 \mu \mathrm{g} / \mathrm{mL}$ streptomycin. All cells were incubated at $37^{\circ} \mathrm{C}$ in a humidified $5 \% \mathrm{CO}_{2}$ atmosphere. 


\section{Cellular uptake}

Intracellular uptake of NPs was evaluated by fluorescence imaging. Activated HSCs were seeded into 6-well plates with the density of $3.0 \times 10^{5}$ cells/well. After $12 \mathrm{~h}$ incubation, cells were treated with ICG-NPs and ICG-RGD-NPs for $4 \mathrm{~h}$. In addition, to further confirm cRGD enhanced cellular uptake, cells were pretreated with free cRGD for 20 min to block $\alpha_{\mathrm{v}} \beta_{3}$ before ICG-RGD-NPs was added. Subsequently, cells were washed with PBS for three times and fixed with $4 \%$ paraformaldehyde for $20 \mathrm{~min}$. After the nuclei were stained with DAPI solution for $15 \mathrm{~min}$, the cells were exposed to a fluorescence microscopy. To survey the time-dependent intracellular accumulation, cells were seeded and incubated as the procedure mentioned above, except cells were treated with ICG-RGD-NPs and incubated for another $2 \mathrm{~h}, 4 \mathrm{~h}, 8 \mathrm{~h}$ and $12 \mathrm{~h}$. Cells were fixed and stained as the above procedure and exposed to a fluorescence microscopy.

\section{Cytotoxicity}

MTT assay was performed to test the cytotoxicity of blank NPs, free GMO, G-RGD-NPs, R-RGD-NPs and G/R-RGD-NPs. First, cells were seeded in 96-well plates at the density of 5000 cells/well. After incubation for $12 \mathrm{~h}$, cells were exposed to $200 \mu \mathrm{L}$ of fresh culture medium containing various formulations of drugs and blank NPs for another $72 \mathrm{~h}$. After that, the culture medium was replaced with $100 \mu \mathrm{L}$ of fresh medium and $20 \mu \mathrm{L}$ of MTT $(5 \mathrm{mg} / \mathrm{mL})$ solution was added afterwards. After incubation for another $4 \mathrm{~h}$, the medium was removed and $200 \mu \mathrm{L}$ DMSO was added. After the crystals were completely dissolved, a microplate reader was used to measure the optical density (OD) at $570 \mathrm{~nm}$. The cell viability was obtained using the following equation:

$$
\text { Cell viability } \%=\frac{O D_{\text {sample }}-O D_{\text {blank }}}{O D_{\text {control }}-O D_{\text {blank }}} \times 100 \%
$$

where $\mathrm{OD}_{\text {sample }}$ represents the $\mathrm{OD}$ value of cells incubated with various drug formulations, $\mathrm{OD}_{\text {control }}$ represents the OD value of cells incubated with complete culture alone, and $\mathrm{OD}_{\text {blank }}$ represents the $\mathrm{OD}$ value of complete culture without cells.

\section{Immunofluorescence and immunohistochemistry}

HSCs were seeded on glass coverslips and incubated for $24 \mathrm{~h}$. Cells were treated with free GMO, G-RGD-NPs, R-RGD-NPs and G/R-RGD-NPs for $6 \mathrm{~h}$. After that, the cells were washed with PBS for $15 \mathrm{~min}$ and fixed in $4 \%$ paraformaldehyde for $30 \mathrm{~min}$. Cells were then washed and permeabilized with $0.1 \%(\mathrm{v} / \mathrm{v})$ Triton X-100 for $20 \mathrm{~min}$ and washed with PBS for $15 \mathrm{~min}$. Then, cells were immersed into $3 \% \mathrm{H}_{2} \mathrm{O}_{2}$ solution and incubated for $10 \mathrm{~min}$ at room temperature to block endogenous peroxidase. After washing with PBS for $15 \mathrm{~min}, 5 \%$ (v/v) bovine serum albumin (BSA) was used as blocking solution for $20 \mathrm{~min}$, followed incubating cells with primary antibodies in blocking solution overnight at $4{ }^{\circ} \mathrm{C}$. Cells were then washed with blocking solution and incubated with fluorophore-labeled secondary antibodies for $50 \mathrm{~min}$ at room temperature. After another washing step, the cells were stained with DAPI for $10 \mathrm{~min}$. After a final washing step, fluorescence images were observed by a fluorescence microscopy. Immunohistochemical analysis of cells was performed by the method mentioned above, except secondary antibodies used instead of fluorophorelabeled secondary antibodies.

\section{Western blot}

Activated HSCs were seeded in 6-well plates with a density of $3.0 \times 10^{5}$ cells/well and incubated for $12 \mathrm{~h}$. Cells were treated with different formulations for $24 \mathrm{~h}$ before western blot analysis. Cells or liver tissues were washed with PBS for three times, followed by protein isolation with radioimmunoprecipitation assay (RIPA) buffer and determination with a BCA protein assay kit. The cell lysates were then mixed with loading buffer, boiled buffer at $100{ }^{\circ} \mathrm{C}$ for $5 \mathrm{~min}$, loaded on the wells of SDS-PAGE gel, transferred by electroporation to poly-(vinylidene difluoride) (PVDF) membrane, incubated with blocking buffer for $30 \mathrm{~min}$ at $37^{\circ} \mathrm{C}$, and cultured with primary antibodies recognizing type I Collagen as well as $\beta$-actin primary antibody overnight at $4{ }^{\circ} \mathrm{C}$ with shaking; secondary antibodies were added and incubated for $4 \mathrm{~h}$ at room temperature followed by analysis using Chemiluminescent substrate. Blots performed in quintuplicate were imaged and quantified using ImageJ software with densitometry analysis.

\section{Liver fibrosis mice model}

Eight-week-old male C57BL/6 mice were purchased from Model Animal Research Center of Nanjing University and reared in specific pathogen-free conditions. The protocol was proved by the Committee on the Ethics of Animal Experiments of Nanjing Medical University. Mice were treated weekly with $\mathrm{CCl}_{4}$ at a dose of $100 \mathrm{mg} /$ $\mathrm{kg}$ (prepared with olive oil 1:1 v/v). After 4 weeks, animals developed liver fibrosis as validated by pathological analysis.

\section{Biodistribution}

Liver fibrosis models were established as described above. Healthy or fibrotic mice were randomly divided into three groups: ICG-NPs and ICG-RGD-NPs at an equivalent dose of $2 \mathrm{mg} / \mathrm{kg}$ ICG with saline as control. In $6 \mathrm{~h}, 12 \mathrm{~h}$ and $24 \mathrm{~h}$ later, mice were sacrificed and major organs including heart, liver, lung, spleen and 
kidney were collected. The tissue fluorescent images were obtained using a Maestro imaging system.

\section{Therapeutic efficiency}

To determine the therapeutic effect, fibrotic mice were divided into five groups and administered intravenously with saline, free GMO, G-RGD-NPs, R-RGD-NPs and G/R-RGD-NPs twice a week for 2 weeks. The dosages of GMO and RNA were $8 \mathrm{mg} / \mathrm{kg}$ and $0.5 \mathrm{mg} / \mathrm{kg}, 16 \mathrm{mg} /$ $\mathrm{kg}$ and $1 \mathrm{mg} / \mathrm{kg}$ and $32 \mathrm{mg} / \mathrm{kg}$ and $2 \mathrm{mg} / \mathrm{kg}$, respectively. During this period, survival rate was $100 \%$ in all groups of mice. After treatment, mice were sacrificed and livers were harvested and stored for further evaluation.

\section{Hematoxylin-Eosin (H\&E), masson and sirius red staining}

To assess the therapeutic effects of various therapy formulations, liver tissues were collected from treated fibrotic mice or healthy mice and fixed with formalin solution (4\%, w/v). H\&E, Masson and Sirius Red staining were performed after dehydration, wax dipping, transparency, embedding and $4 \mu \mathrm{m}$ section of liver tissues. Histological analysis was conducted using an optical microscope.

\section{Results and discussion}

Given the overexpression of $\alpha_{v} \beta_{3}$ on activated HSCs during liver injury, cRGD-modified NPs were prepared based on FDA approved poly(lactic-co-glycolic acid)poly(ethylene glycol) (PLGA-PEG) and encapsulate miR29b and GMO for targeted liver fibrosis therapy. This study aims to explore the target ability and therapeutic efficacy of G/R-RGD-NPs in animal models.

\section{Preparation and characterization of NPs}

NPs were prepared by the oil-in-water $(\mathrm{O} / \mathrm{W})$ emulsion method. DLS results were shown in Fig. 1a-f including average size, polydispersity (PDI) and Zeta potential of blank NPs, G/R-NPs and G/R-RGD-NPs. All NPs exhibited uniform size distribution. Blank NPs displaced average size around $164.7 \mathrm{~nm}$ with PDI 0.168. After miR-29b and GMO were loaded, average size of NPs increased to be $231.2 \mathrm{~nm}$ with PDI 0.140 compared with blank NPs, while Zeta potential of G/R-NPs exhibited almost no change compared with blank NPs. The increased particles size was also observed in TEM results in Fig. 1g, h. All NPs showed negative surface charges, which was caused by carboxyl groups of PLGA-PEG$\mathrm{COOH}$. After cRGD was connected to the surface of

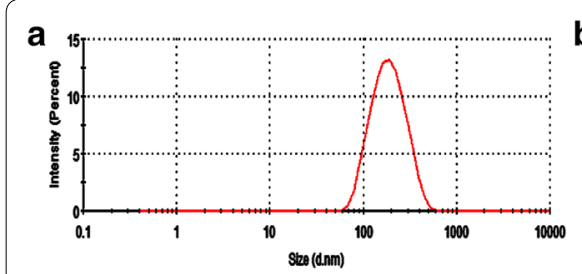

d

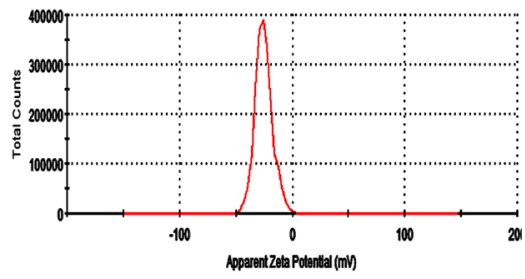

g

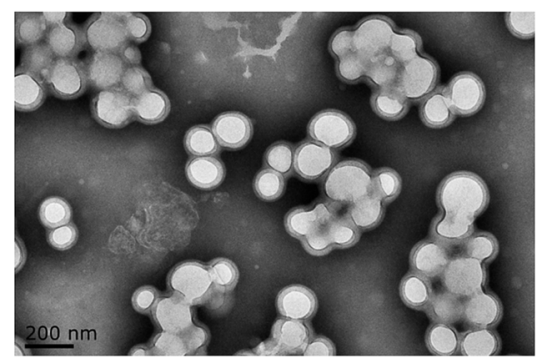

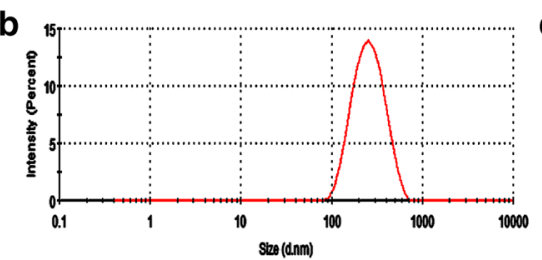

e

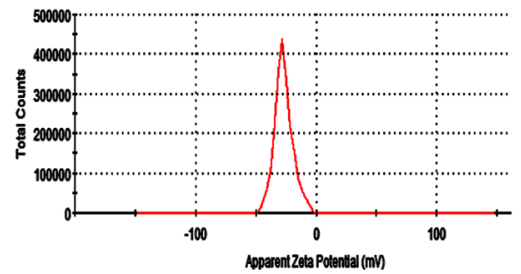

h

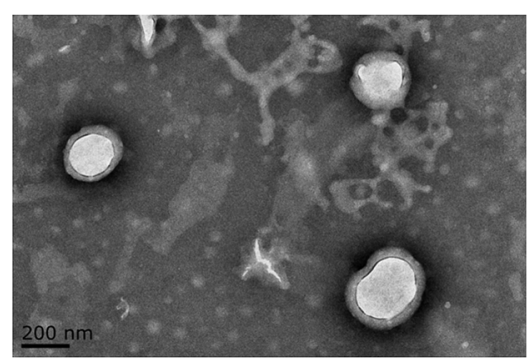

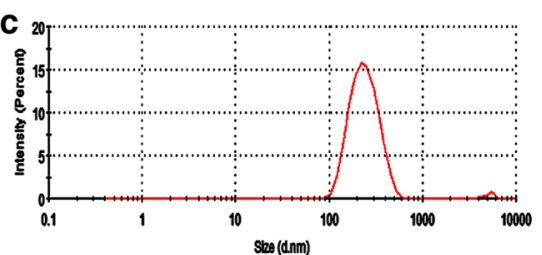

f

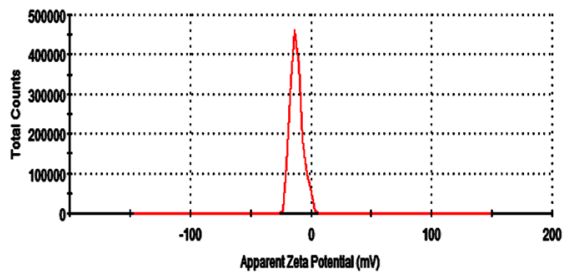

i

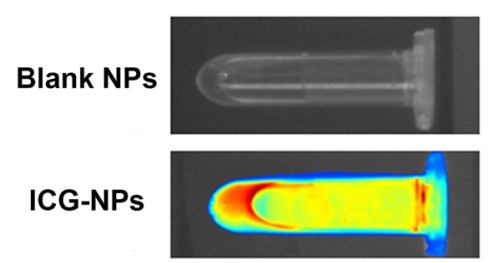

Fig. 1 Size distribution of blank NPs $\mathbf{a}, G / R-N P s \mathbf{b}$ and G/R-RGD-NPs $\mathbf{c}$ measured by DLS; Zeta potential of blank NPs $\mathbf{d}$, G/R-NPs e and G/R-RGD-NPs $\mathbf{f}$ measured by DLS; TEM photos of G/R-NPs $\mathbf{g}$ and G/R-RGD-NPs $\mathbf{h}$; photo of fluorescent images of blank NPs and ICG-RGD-NPs $\mathbf{i}$ using a Maestro imaging system 
NPs, G/R-RGD-NPs displaced average size of $227.2 \mathrm{~nm}$ with PDI 0.195. However, Zeta potential of G/R-RGDNPs changed to be $-12.0 \mathrm{mV}$ compared with G/R-NPs $(-27.0 \mathrm{mV})$, which proved that cRGD was successfully connected to the surface of NPs. The stability of cRGDmodified NPs was measured by DLS. As Additional file 1: Figure S3 depicted, cRGD-modified NPs can keep stable in water and saline, while the average size of cRGDmodified NPs in culture medium with $10 \%$ FBS showed a gradual increase.

DLC and DLE of GMO were measured as $2.5 \%$ and $13.3 \%$, respectively. UV spectra of miR-29b in the supernatant and the initial amount of miR-29b were shown in Additional file 1: Figures S1A, B. After calculation, DLC and DLE of miR-29b were measured as $0.16 \%$ and $61.4 \%$, respectively. It is worth noting that DLE of miR$29 \mathrm{~b}$ was much larger than that of GMO, which is probably determined by the structure of hollow vesicle of NPs. Water-soluble miR-29b was encapsulated in the aqueousfavoring hollow, while lipophilic GMO was loaded in the hydrophobic layer.

The drug release of GMO from NPs was simulated in physiological environment (PBS with $1 \%$ SDS ( $\mathrm{pH} 7.4)$ : methanol $(60 / 40, v / v))$. As shown in Additional file 1: Figure S2, in the first $24 \mathrm{~h}, 77.6 \%$ of GMO was released, while only $6.1 \%$ of GMO was released in the next $24 \mathrm{~h}$. However, almost no miR-29b was detected during $96 \mathrm{~h}$ (data not shown). In the simulated release condition, PEG-PLGA was not degraded and thus the NPs still protected encapsulated drugs in the aqueous phase.

\section{Cellular uptake and cytotoxicity}

It has been reported that $\alpha_{\mathrm{v}} \beta_{3}$ was overexpressed in activated HSCs $[45,46]$. Overexpression of $\alpha_{v} \beta_{3}$ was observed on the activated HSCs compared with LO2 as shown in Additional file 1: Figure S4. Significantly increased green fluorescence was shown on activated HSCs. To demonstrate $\alpha_{v} \beta_{3}$-mediated cellular uptake against activated HSCs, cells were treated with ICG-NPs and ICG-RGD-NPs with or without free cRGD pretreatment. As shown in Fig. 2a, cells treated with ICG-NPs displaced weak red fluorescence after cells incubated with different formulations for $4 \mathrm{~h}$, while cells treated with ICG-RGD-NPs exhibited increased red fluorescence. These results were possibly explained by the fact that cRGD-modified NPs were able to be efficiently internalized into the cells via $\alpha_{v} \beta_{3}$-mediated endocytosis. To further assess the specificity of binding of cRGD-modified NPs to activated HSCs, cells were pretreated with free cRGD to block $\alpha_{v} \beta_{3}$ receptors. As expected, the fluorescence without cRGD pretreatment was much stronger than that with cRGD pretreatment, indicating that the uptake of cRGD-modified NPs can be competed out by excess free cRGD, which was consistent with a previous report [40].

To further demonstrate time-dependent accumulation of cRGD-modified NPs in activated HSCs, cells were treated with ICG-RGD-NPs for $2 \mathrm{~h}, 4 \mathrm{~h}, 8 \mathrm{~h}$ and $12 \mathrm{~h}$. As depicted in Fig. 2b, activated HSCs exhibited increased fluorescence intensity after treated with ICG-RGD-NPs for $12 \mathrm{~h}$. All these results indicated time-dependent accumulation of cRGD-modified NPs.

As shown in Fig. 2c, blank NPs exhibited no cytotoxicity against activated HSCs and $\mathrm{LO} 2$ even at a high concentration of $1 \mathrm{mg} / \mathrm{mL}$. Cell viability of R-RGD-NPs was $78.3 \%$ and $89.4 \%$ after incubation for $72 \mathrm{~h}$ and $48 \mathrm{~h}$, respectively, while cell viability of R-RGD-NPs was over 93\% after incubation for $24 \mathrm{~h}$. Besides, R-RGD-NPs exhibited almost no cytotoxicity against LO2 cells.

The cytotoxicity was evaluated after activated HSCs and LO2 were treated with free GMO, G-RGD-NPs and G/R-RGD-NPs. As shown in Fig. 2d, e, all GMO formulations displayed dose-dependent cytotoxicity against activated HSCs and LO2. G-RGD-NPs exhibited considerable cytotoxicity compared with free GMO against HSCs, while lower cytotoxicity than free GMO especially at high concentration of GMO against LO2. One of the possible reasons to explain these results is that $\alpha_{v} \beta_{3}$ was overexpressed in activated HSCs, facilitating cRGDmodified NPs to be internalized into cells. Besides, it was worth noting that G/R-RGD-NPs exhibited higher cytotoxicity than other formulas at all tested concentrations against HSCs, while considerable cytotoxicity against LO2, which was in accordance with the results in Fig. 2c.

\section{Collagen secretion in activated HSCs}

Collagen plays a vital role in biological process. They served as a structural matrix for tissue maintenance, development and regeneration. Collagen is the most abundant protein in mammals and a main component of ECM. Normal tissue development involves dynamic collagen remodeling processes which was a balance between collagen production and degradation. If the balance was broken, it will cause structural and metabolic abnormalities of collagen and various pathological conditions, including liver fibrosis. Therefore, detecting collagen expression was significant to diagnose and attenuate liver fibrosis. After staining collagen I, the green fluorescence was lower than that of control group after cells were treated with different formulations in Fig. 3a. It can be easily observed that the green fluorescence was the least in activated HSCs treated with G/R-RGD-NPs, indicating enhanced inhibition of collagen production, which was also proved by the results of immunohistochemistry. The collagen abundance was further determined by Western 


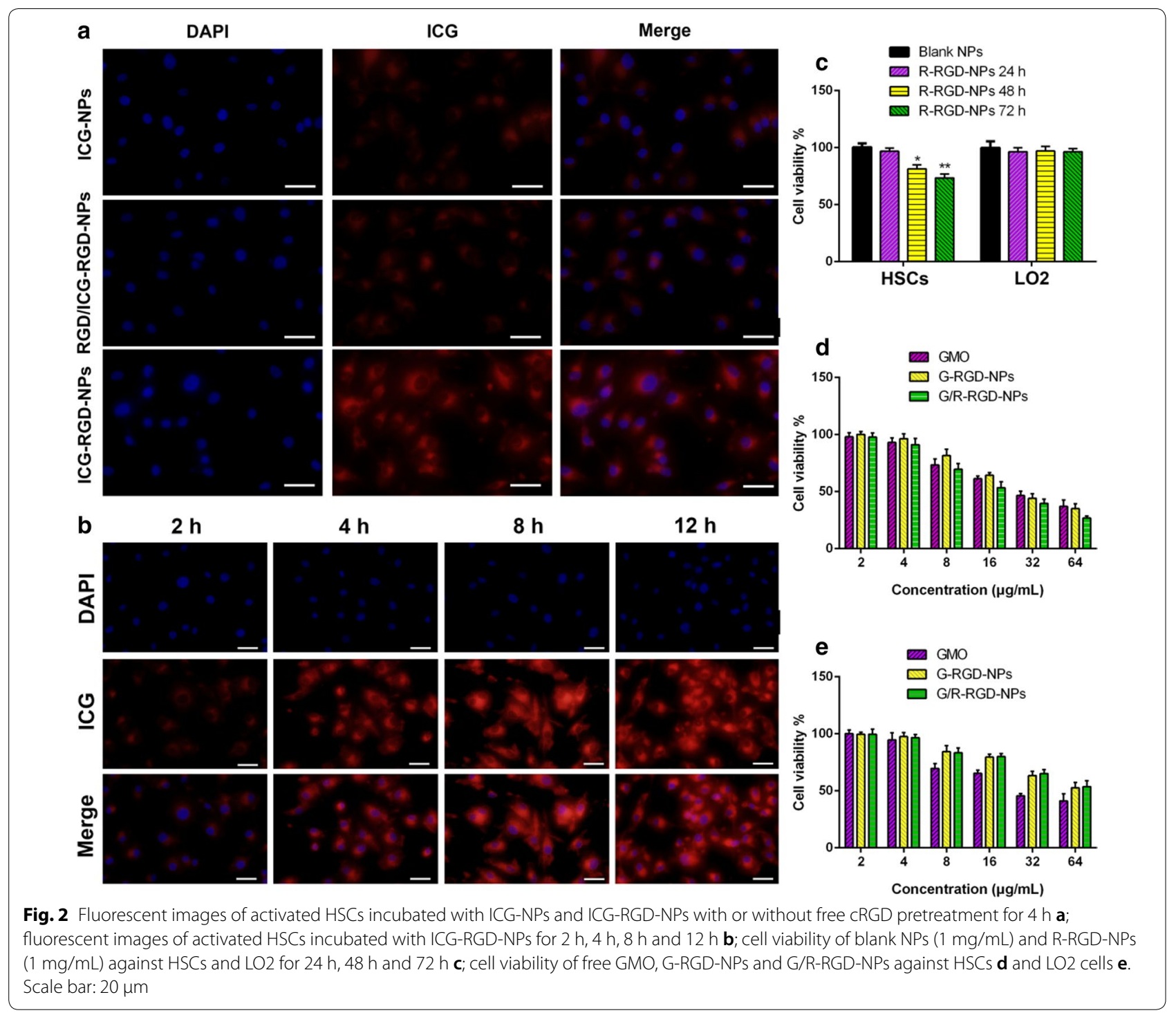

blot as shown in Fig. 3b, c. It was found that collagen was significantly lower than other groups when cells were treated with G-RGD-NPs or G/R-RGD-NPs. Besides, G/R-RGD-NPs exhibited enhanced inhibition of expression of type I collagen compared with G-RGD-NPs, suggesting great potential of G/R-RGD-NPs for liver fibrosis therapy.

\section{Biodistribution and anti-fibrotic effect}

To evaluate biodistribution, fibrotic model mice were administrated with saline, ICG-NPs and ICG-RGDNPs. Saline treatment was conducted as control groups. After $6 \mathrm{~h}, 12 \mathrm{~h}$ and $24 \mathrm{~h}$, mice were sacrificed to collect isolated tissues, including heart, liver, spleen, lung and kidney. The ex vivo imaging of isolated tissues from liver fibrotic was carried out by an imaging system. ICG fluorescence was mainly concentrated in livers. ICG fluorescence was weak at $6 \mathrm{~h}$, while it increased at $12 \mathrm{~h}$ (Additional file 1: Figure S5A). Notably, ICG-RGDNPs exhibited significantly higher fluorescence than ICG-NPs at $12 \mathrm{~h}$ and $12 \mathrm{~h}$ (Fig. 4a).The fluorescence in spleen, lung and kidney increased at $24 \mathrm{~h}$, indicating metabolism of the nanoparticles. Besides, stronger fluorescence was observed in livers from fibrotic or healthy mice treated with ICG-RGD-NPs than those from mice treated with ICG-NPs. Specifically, red fluorescence intensity was slightly higher in livers from healthy mice treated with ICG-RGD-NPs than that treated with ICG-NPs. All these results indicated that liver fibrotic mice model was established successfully and the targeting ability of cRGD-modified NPs was remarkably enhanced, which was mainly caused by two reasons: (1) HSCs activated and proliferated in the fibrotic liver thus increased binding sites for cRGD; (2) 


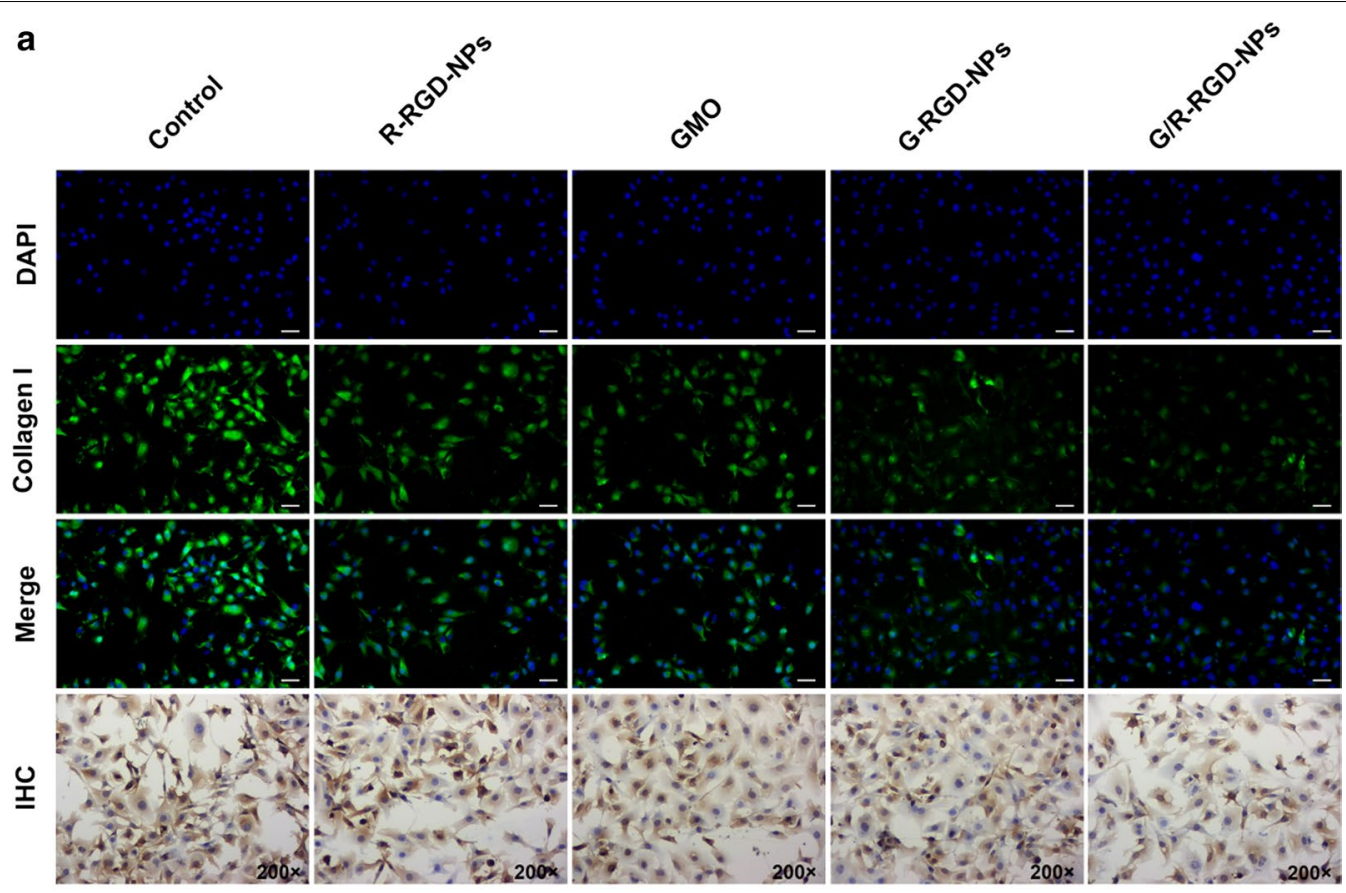

b
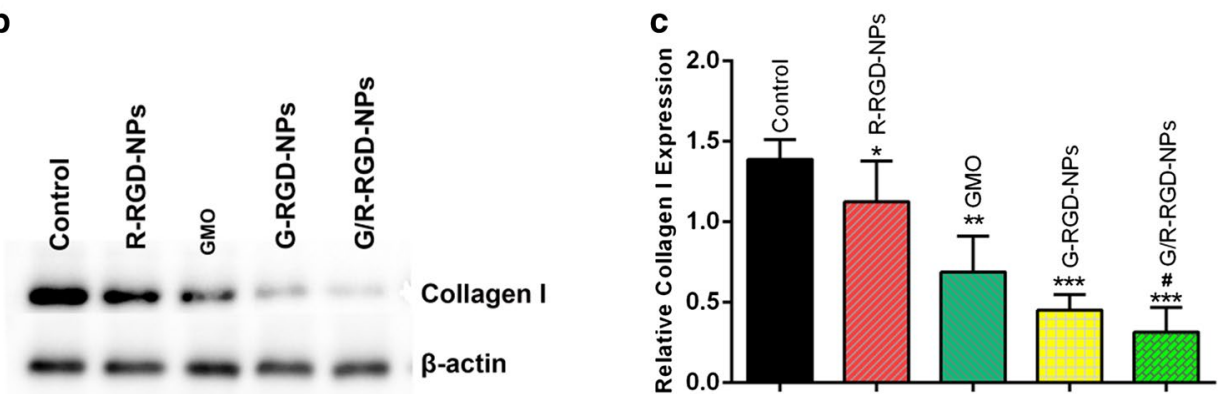

Fig. 3 Immunofluorescence and immunohistochemistry of activated HSCS treated with different drug formulations a; collagen I expression of HSCs treated with different drug formulations $\mathbf{b}$; quantitative analysis of type I collagen in activated HSCs $\mathbf{c} .\left(^{*} p<0.05\right.$, ${ }^{* *} p<0.01$ and ${ }^{* * *} p<0.001$ vs Control; ${ }^{p} p<0.05$ vs G-RGD-NPs)

the fibrotic liver became dysfunctional and thus unable to clear NPs effectively.

To assess the therapeutic effects of various therapeutic formulations, the pathological examination was performed after mice were treated with different therapeutic agents by H\&E, Masson and Sirius Red staining. To search the optimal dosages of GMO and miR-29b for the best therapeutic effect, low, medium and high dose of G/R-RGD-NPs were injected intravenously and the results were shown in Additional file 1: Figure S5B and C. All three groups of G/R-RGD-NPs exhibited dosedependent anti-fibrosis effect and no obvious side effects can be detected. We chose medium dose for the following experiments. In Fig. 4b, H\&E, Masson and Sirius Red staining revealed that treatment of $\mathrm{CCl}_{4}$ induced serious liver fibrosis compared with these of control group. And treatment of R-RGD-NPs displayed slightly anti-fibrotic effect in mice of liver fibrosis. GMO and G-RGD-NPs exhibited better therapeutic effect compared with R-RGD-NPs, and G/R-RGD-NPs exhibited the best antifibrotic effect in mice of liver fibrosis. Type I collagen from collected livers was measured as shown in Fig. 4c, d. Collagen I significantly decreased after mice with liver fibrosis were treated with all therapeutic formulations. GMO and G-RGD-NPs exhibited better inhibition of collagen I production than R-RGD-NPs. And G/R-RGDNPs showed better inhibition of collagen I production than GMO and G-RGD-NPs.

\section{Conclusion}

In summary, G/R-RGD-NPs have been established and applied in mice liver fibrosis model for the treatment of liver fibrosis. ICG-labeled RGD-NPs exhibited selective and time-dependent internalization in activated HSCs. 


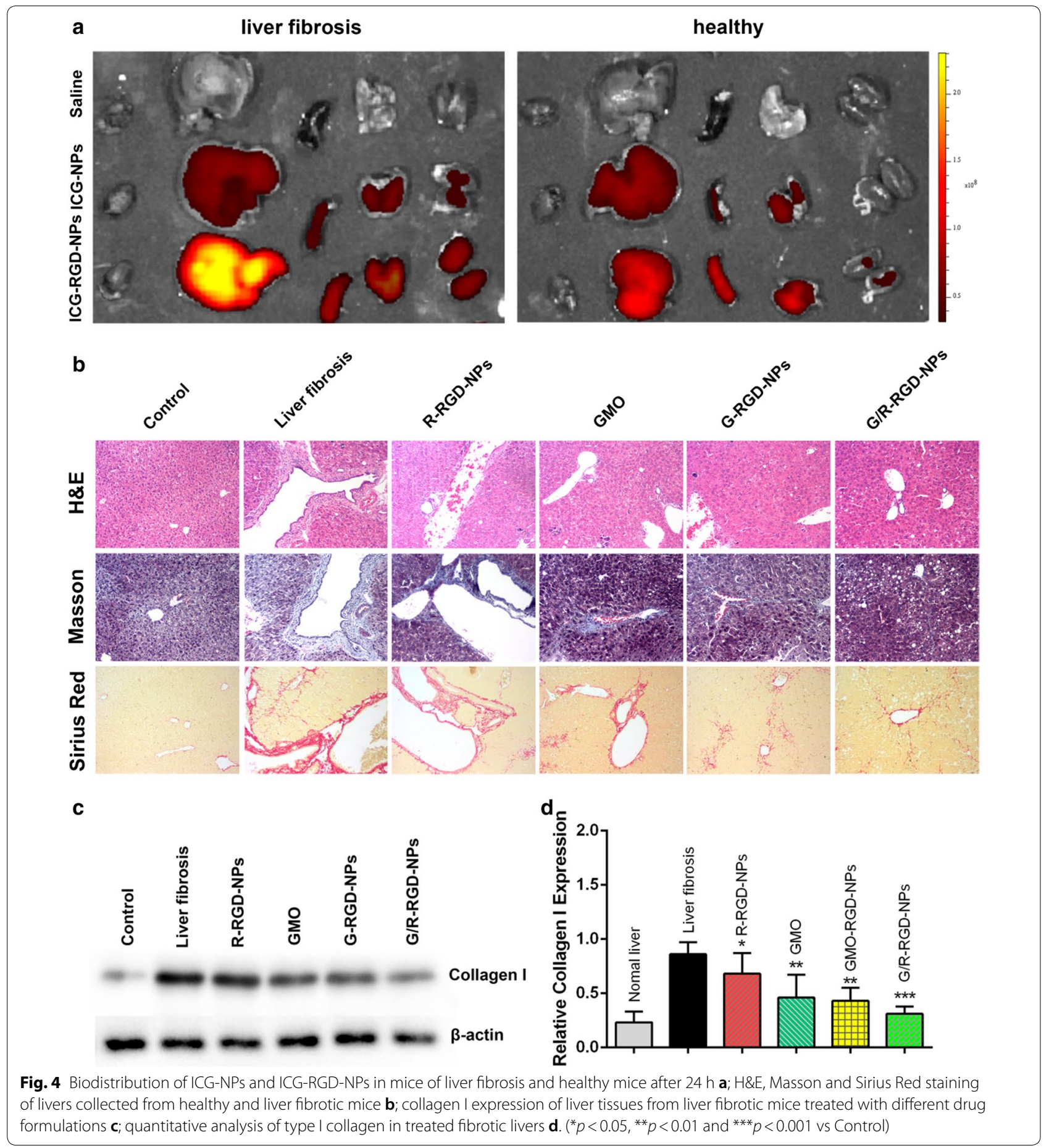

Besides, the targeting ability of NPs was further enhanced by cRGD modification in liver fibrosis mice model. G/RRGD-NPs exhibited higher cytotoxicity on HSCs and enhanced inhibition of protein production compared with free GMO, G-RGD-NPs and R-RGD-NPs. G/RRGD-NPs also exhibited great anti-fibrosis effect on mice model. G/R-RGD-NPs may serve as a novel and effective clinical treatment option for liver fibrosis. 


\section{Supplementary information}

Supplementary information accompanies this paper at https://doi. org/10.1186/s12951-020-00645-y.

Additional file 1: Figure S1. UV spectra of miR-29b in the supernatant (A) and the initial amount of miR-29b (B). Figure S2. Standard curve of GMO (A); drug release of GMO from NPs simulated in physiological environment (PBS, pH 7.4) (B). Figure S3. Stability of CRGD-modified PEG-PLGA NPs in water, saline and culture medium with $10 \%$ FBS. Figure S4. Overexpression of $a_{v} \beta_{3}$ against the activated HSCs compared with LO2. Figure S5. Biodistribution of ICG-NPS and ICG-RGD-NPs in mice of liver fibrosis after 6 $\mathrm{h}$ and $12 \mathrm{~h}(\mathrm{~A})$. H\&E, Masson and Sirius Red staining (B), collagen I expression (C) and quantitative analysis of collagen I (D) of livers collected from liver fibrotic mice treated with G/R-RGD-NPs of low, medium and high dose $\left({ }^{*} p<0.05\right.$ vs Medium dose, $\# p<0.05$ vs Low dose, $\$ p<0.05$ vs Liver fibrosis).

\section{Abbreviations}

HSCs: Hepatic stellate cells; ECM: Extracellular matrix; GMO: Germacrone; NPs: Nanoparticles; PEG-PLGA: Poly(ethylene glycol)-block-poly(lactide-co-glycolide); CRGDfK: Cyclic RGD peptides; H\&E: Hematoxylin-eosin; miRNAs: MicroRNAs; G/R-NPs: GMO- and miR-29b-loaded PEG-PLGA-COOH NPs; G/R-RGD-NPs: CRGDmodified G/R-NPs; R-RGD-NPs: miR-29b-loaded CRGD-modified NPs; G-RGD-NPs: GMO-loaded CRGD-modified NPs; ICG-RGD-NPs: ICG-loaded CRGD-modified NPS; ICG-NPs: ICG-loaded NPs; DLC: Drug loading content; DLE: Efficiency.

\section{Acknowledgements}

We are grateful for the financial support from the National Key R\&D Program of China (2018YFC1707000), National Natural Science Foundation of China (81973483, 81673598, 81473349), The Natural Science Foundation of the Jiangsu Higher Education Institutions of China (18KJB360008), National Standardization Program for Chinese Medicine (ZYBZH-Y-SC-40), Special Fund for Research in the public interest (201507002-2).

\section{Authors' contributions}

DJ wrote the manuscript. QW synthesized Y-PGA-BA. QZ conducted the animal experiments. TL and CJ designed the research plan. HT, MY and MW revised the manuscript. All authors read and approved the final manuscript.

\section{Availability of data and materials}

The datasets used and/or analyzed during the current study are available from the corresponding author on reasonable request.

\section{Ethics approval and consent to participate}

Not applicable.

\section{Consent for publication}

Not applicable.

\section{Competing interest}

The authors declare no conflict of interest.

\begin{abstract}
Author details
1 School of Pharmacy, Nanjing University of Chinese Medicine, Nanjing 210023, China. ${ }^{2}$ Molecular Pharmacology Research Center, School of Pharmaceutical Science, Wenzhou Medical University, Wenzhou 325035, China. ${ }^{3}$ Biomedical Collaborative Innovation Center of Zhejiang, Wenzhou 325035, China. ${ }^{4}$ Affiliated Hospital of Integrated Traditional Chinese and Western Medicine, Nanjing University of Chinese Medicine, Nanjing 210028, China.
\end{abstract}

Received: 10 January 2020 Accepted: 1 June 2020

Published online: 08 June 2020

\section{References}

1. Wilson $C L$, Mann DA, Borthwick LA. Epigenetic reprogramming in liver fibrosis and cancer. Adv Drug Deliv Rev. 2017;121:124-32.

2. Harimoto N, Yoshizumi T, Shimokawa M, Sakata K, Kimura K, Itoh S, Ikegami T, Ikeda T, Shirabe K, Maehara Y. Sarcopenia is a poor prognostic factor following hepatic resection in patients aged 70 years and older with hepatocellular carcinoma. Hepatol. Res. 2016;46:1247-55.

3. Fujita K, Oura K, Yoneyama H, Shi T, Takuma K, Nakahara M, Tadokoro T, Nomura T, Morishita A, Tsutsui K, Himoto T, Masaki T. Albumin-bilirubin score indicates liver fibrosis staging and prognosis in patients with chronic hepatitis C. Hepatol Res. 2019;49:731-42.

4. Yoo W, Lee J, Noh KH, Lee S, Jung D, Kabir MH, Park D, Lee C, Kwon KS, Kim JS, Kim S. Progranulin attenuates liver fibrosis by downregulating the inflammatory response. Cell Death Dis. 2019;10:1-2.

5. Wang Z, Cao D, Li C, Min L, Wang G. Mediator MED23 regulates inflammatory responses and liver fibrosis. PLoS Biol. 2019;17:e3000563.

6. Lee KC, Hsu WF, Hsieh YC, Chan CC, Yang YY, Huang YH, Hou MC, Lin HC. Dabigatran reduces liver fibrosis in thioacetamide-injured rats. Dig Dis Sci. 2019;64:102-12

7. Roeb E. Matrix metalloproteinases and liver fibrosis (translational aspects) Matrix Biol. 2018;68-69:463-73.

8. Baglieri J, Brenner DA, Kisseleva T. The role of fibrosis and liver-associated fibroblasts in the pathogenesis of hepatocellular carcinoma. Int J Mol Sci. 2019;20:1723.

9. Lin TT, Gao DY, Liu YC, Sung YC, Wan D, Liu JY, Chiang T, Wang L, Chen Y. Development and characterization of sorafenib-loaded PLGA nanoparticles for the systemic treatment of liver fibrosis. J Control Release. 2016;221:62-70.

10. El-Mezayen NS, El-Hadidy WF, El-Refaie WM, Shalaby TI, Khattab MM, El-Khatib AS. Hepatic stellate cell-targeted imatinib nanomedicine versus conventional imatinib: a novel strategy with potent efficacy in experimental liver fibrosis. J Control Release. 2017;266:226-37.

11. Zhao Z, Lin CY, Cheng K. siRNA- and miRNA-based therapeutics for liver fibrosis. Transl Res. 2019;214:17-29.

12. Bin Qiao J, Fan QQ, Xing L, Cui PF, He YJ, Zhu JC, Wang L, Pang T, Oh YK, Zhang C, Jiang HL. Vitamin A-decorated biocompatible micelles for chemogene therapy of liver fibrosis. J Control Release. 2018;283:113-25.

13. Liu Y, Wang W, Fang B, Ma F, Zheng Q, Deng P, Zhao S, Chen M, Yang G, He G. Anti-tumor effect of germacrone on human hepatoma cell lines through inducing G2/M cell cycle arrest and promoting apoptosis. Eur J Pharmacol. 2013;698:95-102.

14. Dong Q, Qiu LL, Zhang CE, Chen LH, Feng WW, Ma LN, Yan D, Niu M, Wang JB, Xiao XH. Identification of compounds in an anti-fibrosis Chinese medicine (Fufang Biejia Ruangan Pill) and its absorbed components in rat biofluids and liver by UPLC-MS. J Chromatogr B Anal Technol Biomed Life Sci. 2016;1026:145-51.

15. Su Q, Kumar V, Sud N, Mahato RI. MicroRNAs in the pathogenesis and treatment of progressive liver injury in NAFLD and liver fibrosis. Adv Drug Deliv Rev. 2018;129:54-63.

16. Sekiya Y, Ogawa T, Yoshizato K, Ikeda K, Kawada N. Suppression of hepatic stellate cell activation by microRNA-29b. Biochem Biophys Res Commun. 2011;412:74-9.

17. Wang J, Chu ESH, Chen HY, Man K, Go MYY, Huang XR, et al. microRNA$29 \mathrm{~b}$ prevents liver fibrosis by attenuating hepatic stellate cell activation and inducing apoptosis through targeting PI3KVAKT pathway. Oncotarget. 2015;6:7325-38

18. He $Y$, Huang $C$, peng Zhang $S$, Sun $X$, ran Long $X$, Li J. The potential of microRNAs in liver fibrosis. Cell Signal. 2012;24:2268-72.

19. Ogawa T, lizuka M, Sekiya Y, Yoshizato K, Ikeda K, Kawada N. Suppression of type I collagen production by microRNA-29b in cultured human stellate cells. Biochem Biophys Res Commun. 2010;391:316-21.

20. Chen Q, Gou S, Huang Y, Zhou X, Li Q, Han MK, Kang Y, Xiao B. Facile fabrication of bowl-shaped microparticles for oral curcumin delivery to ulcerative colitis tissue. Colloids Surfaces B Biointerfaces. 2018;169:92-8.

21. Tang B, Zaro JL, Shen Y, Chen Q, Yu Y, Sun P, Wang Y, Shen WC, Tu J, Sun C. Acid-sensitive hybrid polymeric micelles containing a reversibly activatable cell-penetrating peptide for tumor-specific cytoplasm targeting. J Control Release. 2018;279:147-56.

22. Dowaidar M, Abdelhamid HN, Hällbrink M, Zou X, Langel Ü. Graphene oxide nanosheets in complex with cell penetrating peptides for oligonucleotides delivery. Biochim. Biophys. Acta-Gen. Subj. 1861;2017:2334-41. 
23. Ji Y, Lu F, Hu W, Zhao H, Tang Y, Li B, Hu X, Li X, Lu X, Fan Q, Huang W. Tandem activated photodynamic and chemotherapy: Using pH-Sensitive nanosystems to realize different tumour distributions of photosensitizer/ prodrug for amplified combination therapy. Biomaterials. 2019;219:11939.

24. Yang Q, Li P, Ran H, Wan J, Chen H, Chen H, Wang Z, Zhang L. Polypyrrolecoated phase-change liquid perfluorocarbon nanoparticles for the visualized photothermal-chemotherapy of breast cancer. Acta Biomater. 2019;90:337-49.

25. Mu Y, Fu Y, Li J, Yu X, Li Y, Wang Y, Wu X, Zhang K, Kong M, Feng C, Chen X. Multifunctional quercetin conjugated chitosan nano-micelles with P-gp inhibition and permeation enhancement of anticancer drug. Carbohydr Polym. 2019;203:10-8.

26. Li Y, Hu J, Liu X, Liu Y, Lv S, Dang J, Ji Y, He J, Yin L. Photodynamic therapytriggered on-demand drug release from ROS-responsive core-crosslinked micelles toward synergistic anti-cancer treatment. Nano Res. 2019;12:999-1008.

27. Dang J, He H, Chen D, Yin L. Manipulating tumor hypoxia toward enhanced photodynamic therapy (PDT). Biomater Sci. 2017;5:1500-11.

28. Yang Y, Liu J, Liang C, Feng L, Fu T, Dong Z, et al. Nanoscale metal-organic particles with rapid clearance for magnetic resonance imaging-guided photothermal therapy. ACS Nano. 2016;16:2774-81.

29. Zheng Y, Wang W, Zhao J, Wu C, Ye C, Huang M, et al. Preparation of injectable temperature-sensitive chitosan-based hydrogel for combined hyperthermia and chemotherapy of colon cancer. Carbohydr Polym. 2019;22:115039.

30. Liu Y, Xi Y, Zhao J, Zhao J, Li J, Huang G, et al. Preparation of therapeuticladen konjac hydrogel for tumor combination therapy. Chem Eng J. 2019;375:122048.

31. Dowaidar M, Nasser Abdelhamid H, Hällbrink M, Langel Ü, Zou X. Chitosan enhances gene delivery of oligonucleotide complexes with magnetic nanoparticles-cell-penetrating peptide. J Biomater Appl. 2018;33:392-401.

32. Dowaidar M, Abdelhamid HN, Hällbrink M, Freimann K, Kurrikoff K, Zou $X$, et al. Magnetic nanoparticle assisted self-assembly of cell penetrating peptides-oligonucleotides complexes for gene delivery. Sci Rep. 2017;7:1-11.

33. Yang $Y$, Zhu W, Dong Z, Chao Y, Xu L, Chen M, et al. 1D coordination polymer nanofibers for low-temperature photothermal therapy. Adv Mater. 2017;29:1-12.

34. Ergen C, Niemietz PM, Heymann F, Baues M, Gremse F, Pola R, van Bloois L, Storm G, Kiessling F, Trautwein C, Luedde T, Lammers T, Tacke F. Liver fibrosis affects the targeting properties of drug delivery systems to macrophage subsets in vivo. Biomaterials. 2019;206:49-60.

35. Wang M, Zhang M, Fu L, Lin J, Zhou X, Zhou P, Huang P, Hu H, Han Y. Livertargeted delivery of TSG-6 by calcium phosphate nanoparticles for the management of liver fibrosis. Theranostics. 2020;10:36.
36. Li W, Zhou C, Fu Y, Chen T, Liu X, Zhang Z, Gong T. Targeted delivery of hyaluronic acid nanomicelles to hepatic stellate cells in hepatic fibrosis rats. Acta Pharm Sin B. 2019;10:693-710.

37. Fan QQ, Zhang CL, Qiao JB, Cui PF, Xing L, Oh YK, Jiang HL. Extracellular matrix-penetrating nanodrill micelles for liver fibrosis therapy. Biomaterials. 2020;230:119616.

38. Luo J, Zhang P, Zhao T, Jia M, Yin P, Li W, et al. Golgi apparatus-targeted chondroitin-modified nanomicelles suppress hepatic stellate cell activation for the management of liver fibrosis. ACS Nano. 2019;13:3910-23.

39. Qiao J, Fan Q, Zhang C, Lee J, Byun J, Xing L. Hyperbranched lipoid-based lipid nanoparticles for bidirectional regulation of collagen accumulation in liver fi brosis. J Control Release. 2020;321:629-40.

40. Li Y, Pu S, Liu Q, Li R, Zhang J, Wu T, et al. An integrin-based nanoparticle that targets activated hepatic stellate cells and alleviates liver fibrosis. J Control Release. 2019;303:77-90.

41. Lin L, Gong H, Li R, Huang J, Cai M, Lan T, et al. Nanodrug with ROS and $\mathrm{pH}$ dual-sensitivity ameliorates Liver fibrosis via multicellular regulation. Adv Sci. 2020;1903138:1-15.

42. Li C, Zhang X, Chen Q, Zhang J, Li W, Hu H, Zhao X, Qiao M, Chen D. Synthetic polymeric mixed micelles targeting lymph nodes trigger enhanced cellular and humoral immune responses. ACS Appl Mater Interfaces. 2018;10:2874-89.

43. Ma Y, Fan X, Li L. PH-sensitive polymeric micelles formed by doxorubicin conjugated prodrugs for co-delivery of doxorubicin and paclitaxel. Carbohydr Polym. 2016;137:19-29.

44. Luo J, Meng X, Su J, Ma H, Wang W, Fang L, et al. Biotin-modified Polylactic-co-Glycolic acid nanoparticles with improved antiproliferative activity of 15,16-Dihydrotanshinone i in human cervical cancer cells. J Agric Food Chem. 2018;66:9219-30.

45. Li F, Yan H, Wang J, Li C, Wu J, Wu S, Rao S, Gao X, Jin Q. Non-invasively differentiating extent of liver fibrosis by visualizing hepatic integrin av $\beta 3$ expression with an MRI modality in mice. Biomaterials. 2016;102:162-74.

46. Yang J, Hou Y, Ji G, Song Z, Liu Y, Dai G, Zhang Y, Chen J. Targeted delivery of the RGD-labeled biodegradable polymersomes loaded with the hydrophilic drug oxymatrine on cultured hepatic stellate cells and liver fibrosis in rats. Eur J Pharm Sci. 2014;52:180-90.

\section{Publisher's Note}

Springer Nature remains neutral with regard to jurisdictional claims in published maps and institutional affiliations.
Ready to submit your research? Choose BMC and benefit from:

- fast, convenient online submission

- thorough peer review by experienced researchers in your field

- rapid publication on acceptance

- support for research data, including large and complex data types

- gold Open Access which fosters wider collaboration and increased citations

- maximum visibility for your research: over $100 \mathrm{M}$ website views per year

At BMC, research is always in progress.

Learn more biomedcentral.com/submissions 